See Article page 1473.

\section{Commentary: Frailty measures in cardiac surgery: The theory- practice gap}

\author{
Maria Servito, BHSc, Dustin Tanaka, and \\ Bobby Yanagawa, MD, PhD
}

Lim and colleagues ${ }^{1}$ have developed an objective measurement of frailty in octogenarians undergoing cardiac surgery. They show that the $5-\mathrm{m}$ walk test (5MWT) is a simple test of frailty that is highly predictive of postoperative complications: gait times $\geq 6.4$ seconds predicted lower survival for up to 1-year postoperation compared with faster gait speeds. ${ }^{1}$ Slower gait speeds were also associated with higher incidence of delirium, prolonged ventilation and/or reintubation, and need for rehabilitative care at discharge. ${ }^{1}$

Frailty and its importance in medical and surgical outcomes are now abundantly clear. ${ }^{2-6}$ In the cardiac surgery literature, there are ample single-center observational studies, ${ }^{3}$ database studies, ${ }^{7,8}$ and meta-analyses ${ }^{9,10}$ using a range of clinical frailty scores to show an association with adverse outcomes above and beyond the eyeball test. ${ }^{11}$ In fact, since approximately 2007 , there has been a year-over-year increase in articles focused on this topic (Figure 1). Yet, to date, few cardiac surgery programs systematically utilize any frailty score for patients being considered for cardiac surgery. There is a theorypractice gap. The potential reasons are several: lack of an established optimal score for the surgical population among the many studied, little mention of frailty scores in clinical practice guidelines, a culture that relies

From the Division of Cardiac Surgery, University of Toronto and St Michael's Hospital, Toronto, Ontario, Canada.

Disclosures: The authors reported no conflicts of interest.

The Journal policy requires editors and reviewers to disclose conflicts of interest and to decline handling or reviewing manuscripts for which they may have a conflict of interest. The editors and reviewers of this article have no conflicts of interest.

Received for publication March 5, 2021; revisions received March 5, 2021; accepted for publication March 5, 2021; available ahead of print March 10, 2021.

Address for reprints: Bobby Yanagawa, MD, PhD, Division of Cardiac Surgery, University of Toronto and St Michael's Hospital, 30 Bond St, 8th Floor, Bond Wing,

Toronto, ON M5B 1W8 Canada (E-mail: yanagawab@smh.ca).

J Thorac Cardiovasc Surg 2023;165:1486-7

$0022-5223 / \$ 36.00$

Copyright (C) 2021 by The American Association for Thoracic Surgery

https://doi.org/10.1016/j.jtcvs.2021.03.017
Check for updates

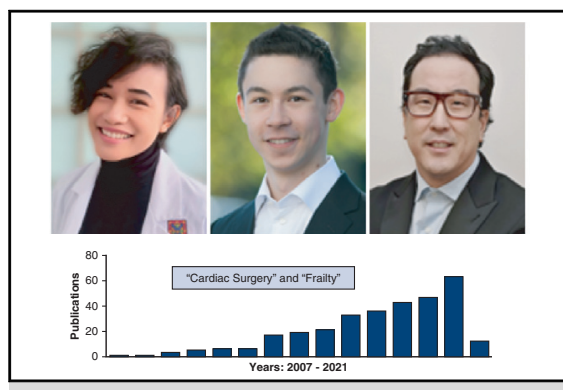

Top, Maria Servito, Dustin Tanaka, and Bobby Yanagawa. Bottom, Cardiac surgery and frailty publications by year.

CENTRAL MESSAGE

The 5-m walk test is a simple test

for identifying frail octogenarians

at risk for adverse outcomes. The

challenge is implementation.

on the eyeball test, the additional time and resources (including specialized equipment or trained personnel) needed, lack of renumeration for determination of frailty scores, and uncertainty of what to do with this information coupled with the lack of large scale, prospective trials on interventions such as perioperative optimization or prehabilitation.

For the 5MWT, many questions remain: Would it be performed by the patient overseen by a resident, nurse specialist, geriatric physician, or surgeon at the time of a surgical consult? What about in-patients awaiting surgery? Should all patients above an age threshold be tested? How about those patients who are young but appear frail?

A change in culture will be necessary to integrate frailty assessment into routine clinical assessment. To this end, frailty can be conceptualized as a vital sign for elderly patients. ${ }^{12}$ Considering that decisions about surgical management of frail patients are often based on a brief history during a huddle or rounds where most of the members will not have physically seen the patient, a frailty score could be of great value. ${ }^{12}$ The Enhanced Recovery After Cardiac Surgery Society has taken a leadership role and recommend prehabilitation for frail patients undergoing elective surgery (Class IIa evidence). We call on all professional societies and funding bodies to put frailty assessment and intervention studies front and center. The science is undisputed, and our patients will surely benefit. 

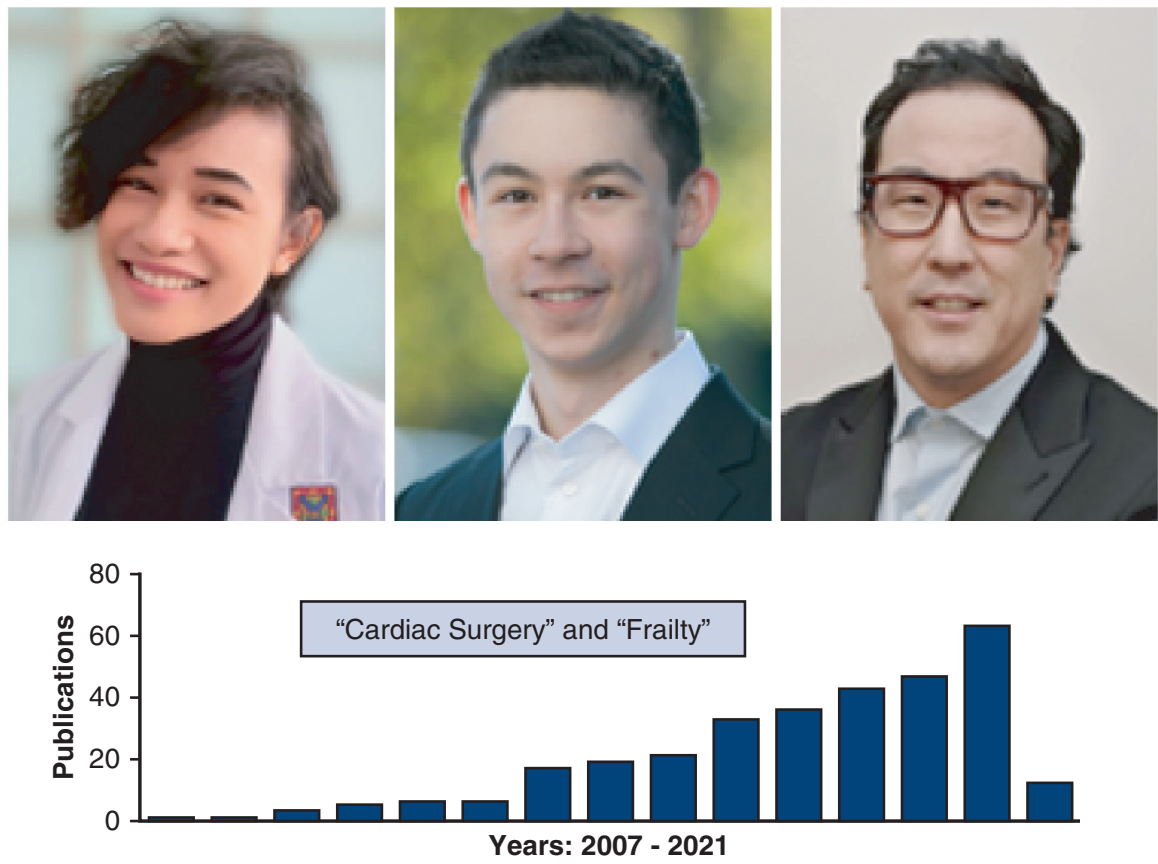

FIGURE 1. Top, Maria Servito, Dustin Tanaka, and Bobby Yanagawa. Bottom, Cardiac surgery and frailty publications by year.

\section{References}

1. Lim S, Jacques F, Babaki S, Babaki Y, Simard S, Kalavrouziotis D, et al. Preoperative physical frailty assessment among octogenarians undergoing cardiac surgery: upgrading the "eyeball" test. J Thorac Cardiovasc Surg. 2023;165:1473-83.e9.

2. Lee DH, Buth KJ, Martin BJ, Yip AM, Hirsch GM. Frail patients are at increased risk for mortality and prolonged institutional care after cardiac surgery. Circulation. 2010;121:973-8.

3. Afilalo J, Mottillo S, Eisenberg MJ, Alexander K, Noiseux N, Perrault L, et al. Addition of frailty and disability to cardiac surgery risk scores identifies elderly patients at high risk of mortality or major morbidity. Circ Cardiovasc Qual Outcomes. 2012;5:222-8.

4. Robinson TN, Wu DS, Pointer L, Dunn CL, Cleveland JC, Moss M. Simple frailty score predicts postoperative complications across surgical specialties. Am J Surg. 2013;206:544-50.

5. Li HC, Wei YC, Hsu R, Chi N, Wang S, Chen Y, et al. Surviving and thriving 1 year after cardiac surgery: frailty and delirium matter. Ann Thorac Surg. 2020; 165:1473-83.

6. Afilalo J, Eisenberg MJ, Morin JF, Bergman H, Monette J, Noiseux N, et al. Gait speed as an incremental predictor of mortality and major morbidity in elderly patients undergoing cardiac surgery. J Am Coll Cardiol. 2010;56:1668-76.
7. Afilalo J, Sharma A, Zhang S, Brennan J, Edwards F, Mack M, et al. Gait speed and 1-year mortality following cardiac surgery: a landmark analysis from the society of thoracic surgeons adult cardiac surgery database. J Am Heart Assoc. 2018;7:e010139.

8. Afilalo J, Lauck S, Kim DH, Lefèvre T, Piazza N, Lachapelle K, et al. Frailty in older adults undergoing Aortic Valve Replacement: The FRAILTY-AVR study. $J$ Am Coll Cardiol. 2017;70:689-700.

9. Sepehri A, Beggs T, Hassan A, Rigatto C, Shaw-Daigle C, Tangri N, et al. The impact of frailty on outcomes after cardiac surgery: A systematic review. $J$ Thorac Cardiovasc Surg. 2014;148:3110-7.

10. Abdullahi YS, Athanasopoulos LV, Casula RP, Moscarelli M, Bagnall M, Ashrafian H, et al. Systematic review on the predictive ability of frailty assessment measures in cardiac surgery. Interact Cardiovasc Thorac Surg. 2017;24: 619-24.

11. Yanagawa B, Graham MM, Afilalo J, Hassan A, Arora RC. Frailty as a risk predictor in cardiac surgery: beyond the eyeball test. J Thorac Cardiovasc Surg. 2018; 156:172-6.e2.

12. Forman DE, Alexander KP. Frailty: a vital sign for older adults with cardiovascular disease. Can J Cardiol. 2016;32:1082-7. 